CERN - Data Handling Division $\mathrm{DD} / 74 / 10$

B. Fornberg

8 May 1974 



\section{ON A FOURIER METHOD FOR THE INTEGRATION OF HYPERBOLIC EQUATIONS}

\section{B. Fornberg}

Data Handling Division, CERN, Geneva, Switzerland

\section{ABSTRACT}

In this paper we consider high-order difference approximations of $\partial / \partial \mathrm{x}$ and especially one method based on the fast Fourier transform. It is used to approximate space derivatives for hyperbolic partial differential equations, and its accuracy, stability and speed on a computer are discussed.

AMS 1970 subject classification: Primary 65M10, Secondary 39A05, $42 \mathrm{~A} 12$.

Key phrases: High-order difference approximations, hyperbolic differential equations, Fourier interpolation, fast Fourier transform (FFT). 



\section{INTRODUCTION}

Consider a first-order hyperbolic system of partial differential equations

$$
\frac{\partial u}{\partial t}=\sum_{v=1}^{s} A_{v}(x, t) \frac{\partial u}{\partial x_{v}}+B(x, t) u+F(x, t),
$$

where $A_{V}$ and $B$ are square $n \times n$ matrices, $u$ and $F$ column vectors of $n$ elements. We assume that the matrices $A_{V}$ are Hermitian and that $A_{V}, B, F$ and the initial conditions for $u_{v}, \nu=1,2, \ldots, n$, are periodic in the space variables $x_{\nu}$, $v=1,2, \ldots, s$.

The Fourier method considered here consists of replacing the $x$-derivatives over a grid with uniform spacing $h$, by the analytical derivative of the interpolating trigonometrical polynomial over the grid points. This can be done by two fast Fourier transforms (FFTs) for each $u_{\nu}, \nu=1,2, \ldots, n$ and each $x_{v}, v=1,2, \ldots, s$. This leads to a system of differential-difference equations which can be solved numerically by introducing a time step $k$ and using for example central difference approximation in time. This method was originally suggested by Kreiss and 01iger [3], and has later been considered by Orszag [4, 5] and the present author $[1]$.

The purpose of this paper is to give a survey of the basic properties of this technique, such as its stability and accuracy. We compare it with high-order difference approximations and give numerical results.

In general, we will not get a stable approximation if we straightforwardly apply the Fourier method to the space derivatives in (1) even if (for theoretical reasons) we keep the derivatives in time. This has been demonstrated for a scalar equation by Kreiss and 0liger [3]. One way to get around this instability is to rewrite (1) in antisymmetric form, as we have done in equation (3) in the next section. However, this rewriting is numerically inefficient since we have to approximate twice as many derivatives (involving $u$ ). We will study a model equation

$$
\frac{\partial u}{\partial t}+a(x) \frac{\partial u}{\partial x}=0
$$

and investigate the behaviour of the Fourier method if it is applied directly without any rewriting. The method turns out to be stable if $a(x) \geq \varepsilon>0$ $[$ or $\mathrm{a}(\mathrm{x}) \leq \varepsilon<0]$.

In Section 3 we repeat briefly the known argument showing the very high formal accuracy of the method, and we indicate how an error estimate can be obtained.

Section 4 contains some basic theory for high-order difference approximations, and we find that the Fourier method can be regarded as a limit of such approximations when their width and accuracy (for a periodic problem with a fixed mesh) tend to infinity. 
In Section 5, finally, we present some numerical results.

\section{STABILITY CONDITIONS}

Assuming that the matrices $A_{v}$ are differentiable with respect to $x_{v}$, we can rewrite (1) in antisymmetric form

$$
\frac{\partial u}{\partial t}=\frac{1}{2} \sum_{V=1}^{S} A_{V} \frac{\partial u}{\partial x_{V}}+\frac{1}{2} \sum_{v=1}^{S} \frac{\partial}{\partial x_{V}}\left(A_{v} u\right)-\frac{1}{2} \sum_{V=1}^{S} \frac{\partial A_{v}}{\partial x_{V}} u+B u+F .
$$

We define the scalar product and the norm by

$$
(f, g)=\iint \ldots \int f^{*} g d x_{1} d x_{2} \ldots d x_{s}
$$

and

$$
\|f\|^{2}=(f, f) .
$$

Each integral is taken over one period of the corresponding space variable, and by $f^{*}$ we mean the vector $f$ transposed and conjugated. We get from (3), after scalar multiplication with $u$ and using partial integration,

$$
\frac{1}{2} \frac{\partial}{\partial t}\|u\|^{2}=\operatorname{Re}\left\{-\frac{1}{2}\left(u, \sum_{\nu=1}^{S} \frac{\partial A}{\partial x_{\nu}} u\right)+(u, B u)+(u, F)\right\} \leq \text { constant } \cdot\|u\|^{2}
$$

assuming that $\partial A_{V} / \partial x_{\nu}, B$ and $F$ are bounded. The solution to (3) increases thus at most exponentially in time. If all space derivatives in (3) are straightforwardly approximated by any antisymmetric difference operator, we find in the same way as above that the obtained differential-difference system is stable in the corresponding discrete norm. This applies in particular to the Fourier method, which later will be shown to be equivalent to certain wide antisymmetric difference operators. The rewriting of equation (1) in the form of equation (3) at least doubles the work, and we want to avoid it.

Let us, instead of the general system (3), consider the model equation (2)

$$
\frac{\partial u}{\partial t}+a(x) \frac{\partial u}{\partial x}=0,
$$

where $u=u(x, t)$ is a scalar function. We consider the period interval $[0,1]$ and discretize it in $2 N+1$ intervals of length $h$. Let $T$ be the operator which corresponds to using the Fourier method or any difference method to find approximations for the derivative at the mesh points, using the values at these points. Since $\mathrm{T}$ is linear, we can represent it by a square matrix $\mathrm{S}$ of order $2 \mathrm{~N}+1$. 
Theorem. Consider the differential-difference scheme obtained when (2) is discretized only in space and approximated in space with any method such that its corresponding matrix $\mathrm{S}$, as defined above, is anti-Hermitian. Assuming that the coefficient $a(x)$ satisfies $a(x) \geq \varepsilon>0$, we have

$$
\|v\|_{t=t_{1}} \leq \sqrt{\frac{\max a(x)}{\min a(x)}}\|v\|_{t=t_{2}},
$$

where $\mathrm{v}$ is a column vector of length $2 \mathrm{~N}+1$ containing the mesh values. The norms are the standard $L_{2}$-vector norms, and $t_{1}$ and $t_{2}$ are any time values.

Proof. The approximation of equation (2) is

$$
\frac{d v}{d t}+A S v=0
$$

where $A$ is a positive definite diagonal matrix with the diagonal elements equal to the values of $a(x)$ at the mesh points. We get

$$
\begin{aligned}
\frac{d}{d t}\left(v, A^{-1} v\right) & =-\left(A S v, A^{-1} v\right)-\left(v, A^{-1} A S v\right) \\
& =-\left(v, S^{*} A^{*} A^{-1} v\right)-(v, I S v)=-\left(v,\left(S^{*}+S\right) v\right)=0 .
\end{aligned}
$$

Hence the solution to $\mathrm{dv} / \mathrm{dt}+\mathrm{ASv}=0$ is constant in time with respect to the $A^{-1}-$ norm $\|v\|_{A^{-1}}^{2}=\left(v, A^{-1} v\right)$. We have

$$
\frac{1}{\max a(x)}(v, v) \leq\left(v, A^{-1} v\right) \leq \frac{1}{\min a(x)}(v, v) \text {, }
$$

from which the theorem follows.

We will now show that the condition that $S$ is anti-Hermitian is satisfied for the Fourier method and for any difference approximation with antisymmetric coefficients. Since the Fourier method treats the first N Fourier components without error, the vectors

$$
v(\omega)=\left(e^{2 \pi i \omega x_{0}}, e^{2 \pi i \omega x_{1}}, \ldots, e^{\left.2 \pi i \omega x_{2 N}\right)^{T}}\right.
$$

[where $\omega$ is an integer, $|\omega| \leq N$ and $x_{\nu}=\nu h, \nu=0,1, \ldots, 2 N$ ] are eigenvectors of $\mathrm{S}$ with the eigenvalues $2 \pi i \omega$, i.e. $\mathrm{Sv}(\omega)=2 \pi i \omega v(\omega)$. [We can also choose the number of points even, $2 \mathrm{~N}$. The eigenvectors are then, apart from $v(\omega),|\omega| \leq N-1$, also $v(N)+v(-N)$ with eigenvalue 0.$]$ Since the eigenvectors are orthogonal and the eigenvalues are purely imaginary, $S$ is an anti-Hermitian matrix. It can easily be verified that the same vectors $\mathrm{v}(\omega)$ also are eigenvectors if we use an arbitrary difference approximation with antisymmetric coefficients. The eigenvalues are again purely imaginary, however depending on the approximation. 


\section{ERROR ESTIMATES}

\subsection{Formal accuracy for space derivatives}

Let us consider a function $v(x)$ which is analytic and has period 1 . We introduce as usual a mesh with spacing $h=1 /(2 \mathrm{~N}+1)$ and want to estimate how fast the error in the Fourier approximation of $\mathrm{v}^{\prime}(\mathrm{x})$ at the mesh points goes to zero when $h \rightarrow 0$ (or equivalently $N \rightarrow \infty$ ). We have

$$
v(x)=\sum_{\omega} \hat{v}(\omega) e^{2 \pi i \omega x}=\sum_{|\omega| \leq N}+\sum_{|\omega|>N}=\Sigma_{1}+\Sigma_{2},
$$

where $|\hat{v}(\omega)|<c_{1} e^{-c_{2}|\omega|}, c_{1}$ and $c_{2}>0$. [This estimate follows from the fact that the Fourier series for $\mathrm{v}(\mathrm{x})$ should be valid also in some neighbourhood of the real axis.] Let $\mathrm{T}$ be the linear operator corresponding to derivation with the Fourier method. We get

$$
\mathrm{v}^{\prime}(\mathrm{x})=\Sigma_{1}^{\prime}+\Sigma_{2}^{\prime}=\mathrm{T} \Sigma_{1}+\Sigma_{2}^{\prime}=\mathrm{T} \Sigma_{1}+\mathrm{T} \Sigma_{2}+\left(\Sigma_{2}^{\prime}-\mathrm{T} \Sigma_{2}\right)=\mathrm{T} \mathrm{v}(\mathrm{x})+\left(\Sigma_{2}^{\prime}-\mathrm{T} \Sigma_{2}\right) .
$$

Since

$$
\Sigma_{2}^{\prime}=\sum_{|\omega|>N} i \omega \hat{v}(\omega) e^{i \omega x}
$$

and

$$
\mathrm{T} \Sigma_{2}=\sum_{|\omega| \leq N} \sum_{k \neq 0} i \omega \hat{v}[\omega+k(2 N+1)] e^{i \omega x}
$$

a straightforward estimate shows that both $\Sigma_{2}^{\prime}$ and $T \Sigma_{2}$ are bounded by a constant times $\mathrm{N} e^{-c_{2} N}$, and thus the error is of the form $\left(c_{3} / h\right) e^{-c_{2} / h}, c_{2}$ and $c_{3}>0$. If we assume $\mathrm{v}(\mathrm{x})$ to be only infinitely differentiable on the real axis instead of analytic, we get similarly that the error decreases faster than any power of $h$.

\subsection{Error estimate for the differential-difference equation}

The analytical solution to (2) can be written

$$
u(x, t)=\sum_{\omega} \hat{u}(\omega, t) e^{2 \pi i \omega x}=\sum_{|\omega| \leq N}+\sum_{|\omega|>N}=u_{N}+R_{N} .
$$

Theorem. The difference between the approximate and exact solution to Eq. (2) can be estimated in the discrete $L_{2}$-norm at $t=t_{1}$ by (assume we started at $t=0$ )

$$
\|v-u\|_{t=t_{I}} \leq\left\|R_{N}\right\|_{t=t_{1}}+\sqrt{\frac{\max a(x)}{\min a(x)}} t_{l}\left\|\frac{\partial R_{N}}{\partial t}+a(x) \frac{\partial R_{N}}{\partial x}\right\|_{\max } \text { in }\left[0, t_{1}\right] \text {. }
$$

Eroof. From equation (4) we get

$$
\frac{\partial u_{N}}{\partial t}=-a(x) \frac{\partial u_{N}}{\partial x}-\frac{\partial R_{N}}{\partial t}-a(x) \frac{\partial R_{N}}{\partial x}=-a(x) T u_{N}-\frac{\partial R_{N}}{\partial t}-a(x) \frac{\partial R_{N}}{\partial x} \text {. }
$$


This is valid for all $\mathrm{x}$, in particular at the mesh points. Letting $\mathrm{u}_{\mathrm{N}}$ and $\mathrm{R}_{\mathrm{N}}$ now denote column vectors and $A$ and $S$ the matrices introduced before, we get

$$
\frac{\partial u_{N}}{\partial t}=-A S u_{N}-\left(\frac{\partial R_{N}}{\partial t}-A \frac{\partial R_{N}}{\partial x}\right) \text {. }
$$

Subtracting

$$
\frac{\partial v}{\partial t}=-A S v
$$

leads to

$$
\frac{\partial}{\partial t}\left(u_{N}-v\right)=-A S\left(u_{N}-v\right)-\left(\frac{\partial R_{N}}{\partial t}-A \frac{\partial R_{N}}{\partial x}\right) .
$$

At $t=0, u_{N}-v$ is zero, and the solution to the homogenous equation can grow at most with the factor $\sqrt{\max a(x) / \min a(x)}$ in the $L_{2}$-norm. This leads to equation (5).

The problem of estimating the error $\|v-u\| t=t_{1}$ is thus reduced to estimating the norms on the right-hand side of (5). These norms depend only on the analytic solution of equation (2), not on the special approximation method used. We will now proceed to give some heuristic arguments which show how these norms can be estimated fairly accurately. We will also see in which way these norms depend on $u(x, 0)$ and $a(x)$.

To equation (2) corresponds a system of eigenfunctions, which are obtained by substituting $u(x, t)=e^{2 \pi i \lambda} \nu^{t} \phi_{\nu}(x)$ in the equation. This gives (after norma1ization)

$$
\phi_{\nu}(\mathrm{x})=\exp \left(-2 \pi i \lambda \int_{0}^{\mathrm{x}} \frac{\mathrm{d} \xi}{\mathrm{a}(\xi)}\right) .
$$

The functions $\phi_{\nu}$ have the period 1. Thus the $\lambda_{\nu}$ are multiples of $\lambda=1 / \int_{0}^{1} \mathrm{~d} \xi / \mathrm{a}(\xi)$. Nothing essential is changed if $a(x)$ is multiplied by a constant factor such that $\lambda=1$. We assume in the rest of this section that this has been done. In these eigenfunctions, which form a complete system and are orthogonal with respect to the scalar product,

$$
(f, g)=\int_{0}^{1} \bar{f} g \frac{1}{a(x)} d x,
$$

we can expand any (piecewise continuous) solution to (2) as

$$
u(x, t)=\sum_{\nu} \hat{u}(\nu) e^{2 \pi i \nu t} \phi_{\nu}(x) .
$$

We note that the coefficients $\hat{u}(\nu)$ in this expansion are time-independent in contrast to the coefficients $\hat{\mathrm{u}}(\omega, t)$ for the usual Fourier expansion, defined in equation (4).

The essential point for the performance of the Fourier method is how many eigenfunctions are well approximated. The characteristic property of the method is that it treats the set of functions $e^{2 \pi i \omega x},|\omega| \leq N$, without error. The ideal 
situation is if these happen to agree with $\phi_{V}(x),|\nu| \leq N$, which actually is the case if we have a constant coefficient problem. Otherwise an eigenfunction is treated well only if the amplitudes of all frequency components present in it, and exceeding $\mathrm{N}$, are small. For a $(\mathrm{x})$ given later in Diagram 4 the 10th, 30th and 50 th eigenfunctions have been evaluated. Figure $I$ shows the amplitudes of different frequency components in these eigenfunctions. We get almost exact symmetry around the frequency that corresponds to the number of the eigenfunction. Numerical tests suggest that the highest frequency that cannot be ignored is roughly this number times $1 / \min a(x)$, this approximation being better the less $a(x)$ deviates from 1 . The smoothness of $a(x)$ seems in this context to be of minor importance.

We shall finally indicate a possible way to estimate the norms on the righthand side of (5). By equation (6) for $t=0$, we can evaluate or estimate $\hat{u}(v)$. Since the magnitudes of the coefficients in $(6),\left|\hat{u}(\nu) e^{2 \pi i v t}\right|$, are time-independent, we need only the estimate just considered of the different Fourier components in the eigenfunctions to obtain a time-independent bound for $|\hat{u}(\omega, t)|$. With this estimate, we can find a bound for $\left\|\mathrm{R}_{\mathrm{N}}\right\|$, and assuming

$$
a(x)=\sum_{\omega} \hat{a}(\omega) e^{2 \pi i \omega x}
$$

a straightforward calculation shows that

$$
\frac{\partial R_{N}}{\partial t}+a(x) \frac{\partial R_{N}}{\partial x}=-\left(\sum_{|\mu|>N} \sum_{\omega}-\sum_{\mu} \sum_{|\omega|>N}\right)\left[2 \pi i \omega \hat{u}(\omega, t) \hat{a}(1 !-\omega) e^{2 \pi i \mu x}\right] .
$$

Most ot the terms cancel and the whole expression can be estimated in terms of $|\hat{u}(\omega, t)|$ and $|\hat{a}(\omega)|$ only. We note that in the case of constant coefficients $|\hat{a}(\omega)|=0$ for $\omega \neq 0$ and everything vanishes.

\section{COMPARISONS WITH DIFFERENCE METHODS}

\subsection{Coefficients for difference approximations of $\partial / \partial x$}

By $I$ we denote the identity operator $\operatorname{Iv}(x)=v(x)$ and by $E$ the translation operator $\operatorname{Ev}(x)=v(x+h)$. By $D_{0}, D_{+}$and $D_{-}$we denote the usual central, forward and backward difference approximations of $\partial / \partial \mathrm{x}$, respectively, i.e. $2 \mathrm{hD} \mathrm{D}_{0}=E-\mathrm{E}^{-1}$, $h D_{+}=E-I$ and $h D_{-}=I-E^{-1}$.

We define the difference approximation

$$
D=\sum_{\nu=-N}^{N} \beta_{\nu} E^{\nu}
$$

of $\partial / \partial x$ to be accurate to the order of $p$ if $p$ is the highest integer for which Dv by Taylor expansion can be represented as $\mathrm{dv} / \mathrm{dx}+0\left(\mathrm{~h}^{\mathrm{p}}\right)$. 
We consider only approximations $D$ which are antisymmetric in the sense that $\beta_{-\nu}=-\beta_{\nu}, \nu=1,2, \ldots, N, \beta_{0}=0$. This antisymmetry is normally necessary for stability (see [2], and it also gives the highest accuracy for a fixed $N$. We can then express $D$ in (7) as

$$
D=D_{0} \sum_{\nu=0}^{N-1}(-1)^{\nu} \alpha_{2 \nu}\left(h^{2} D_{+} D_{-}\right)^{\nu} \text {. }
$$

The optimal choice of $\alpha_{2 \nu}$ concerning accuracy is given by

$$
\begin{aligned}
& \alpha_{0}=1 \\
& \alpha_{2 \nu}=\alpha_{2 \nu-2} \frac{v}{4 v+2}, \quad v=1,2, \ldots .
\end{aligned}
$$

These $\alpha_{2 \nu}$, which are independent of $\mathrm{N}$, make (8) accurate to the order of $2 \mathrm{~N}$. To obtain the relations ( 9 ), we apply ( 8 ) to the function $e^{2 \pi i \omega x}$, i.e.

$$
D e^{2 \pi i \omega x}=\frac{i}{h} \sin 2 \pi \omega h \sum_{\nu=0}^{N-1} \alpha_{2 \nu^{2}}{ }^{2 \nu}(\sin \pi \omega h)^{2 \nu} e^{2 \pi i \omega x} .
$$

We want the coefficient for $e^{2 \pi i \omega x}$ on the right-hand side above to converge to $2 \pi i \omega$ when $N$ increases to infinity, i.e. to find constants $\alpha_{2 v}$ such that

$$
2 \pi i \omega=\frac{i}{h} \sin 2 \pi \omega h \sum_{\nu=0}^{\infty} \alpha_{2 v^{2}}{ }^{2 \nu}(\sin \pi \omega h)^{2 \nu} \text {. }
$$

Putting $\xi=\pi \omega$ h we get

$$
2 \xi=\sin 2 \xi \sum_{\nu=0}^{\infty} \alpha_{2 \nu^{2}}{ }^{2 \nu}(\sin \xi)^{2 \nu}
$$

The substitution $t=\sin \xi$ leads to

$$
\frac{\arcsin t}{\sqrt{1-t^{2}}}=\sum_{\nu=0}^{\infty} \alpha_{2 \nu} 2^{2 \nu} t^{2 \nu+1}
$$

The relations (9) follow from the known Taylor expansion of $(\arcsin t) / \sqrt{1-t^{2}}$. The fact that the coefficients $\alpha_{2 \nu}$ are optimal also for a finite $N$ may be deduced from the fact that a truncated Taylor expansion is the unique polynomial which gives, for a fixed degree, the highest order of fitting around the point of expansion.

The formula (8) with $\alpha_{2 v}$ given by (9) may be expressed in the form

$$
D=\frac{1}{2 h} \sum_{\nu=-N}^{N} \beta_{\nu, N} E^{\nu} \nu(x) \text {. }
$$

In this representation, the coefficients depend on both $\nu$ and $N$ :

$$
\beta_{\nu, N}=\frac{2(N !)^{2}(-1)^{\nu+1}}{\nu(N+\nu) !(N-\nu) !}, \quad \nu \neq 0, \quad \beta_{O, N}=0,
$$


Hence, $A / \nu$ can for each method be expressed as a function of TV/e. Figure 2 shows this relation. For the Fourier method, however, $A / \nu$ is not a function of $T \nu / e$ but of $v$ only. It is therefore given as different lines for some values of $\nu$. The FFT routine used is described in [1]. We see from the diagram that the secondorder method is completely inferior to higher-order methods, which is also pointed out in [3]. We can also see that not much is to be gained by accuracies higher than sixth order. A detailed study along similar lines to this has been done recently by Swartz and Wendroff [6].

In reality, one has to discretize also in the time direction, and normally, leap-frog with a small step is the best one can do. The efficiency comparisons between the different methods for fixed time and accuracy are now also valid. For reasons of accuracy, the time step must be very small, and the stability condition

$$
\lambda=\frac{\mathrm{k}}{\mathrm{h}}<\frac{1}{\max _{\xi} \mid \mathrm{f}(\mathrm{N}, \xi) T}
$$

is no problem. For the Fourier method we have similarly $\lambda<(2 \mathrm{~N}+1) / 2 \pi N$ assuming $2 \mathrm{~N}+1$ points in the space direction. The critical values of $\lambda$ are given in Tables 1 and 3 .

\section{NUMERICAL RESULTS}

\subsection{Eigenvalues for one-dimensional examples}

The matrices AS, discussed in the stability proof in Section 2, can be explicitly written down, and the eigenvalues (and eigenvectors) evaluated. Since all eigenvalues for the analytic solution are multiples of the first one, this gives us a way to check how many eigenfunctions are treated well by the Fourier method. The eigenvalues corresponding to the three functions $a(x)$ in Diagrams $2=3$ and 4 are evaluated below by the $Q R-m e t h o d$ on the matrices AS. The space interval $[0,1]$ was discretized by 27 mesh points, i.e. 13 non-trivial frequencies were used.
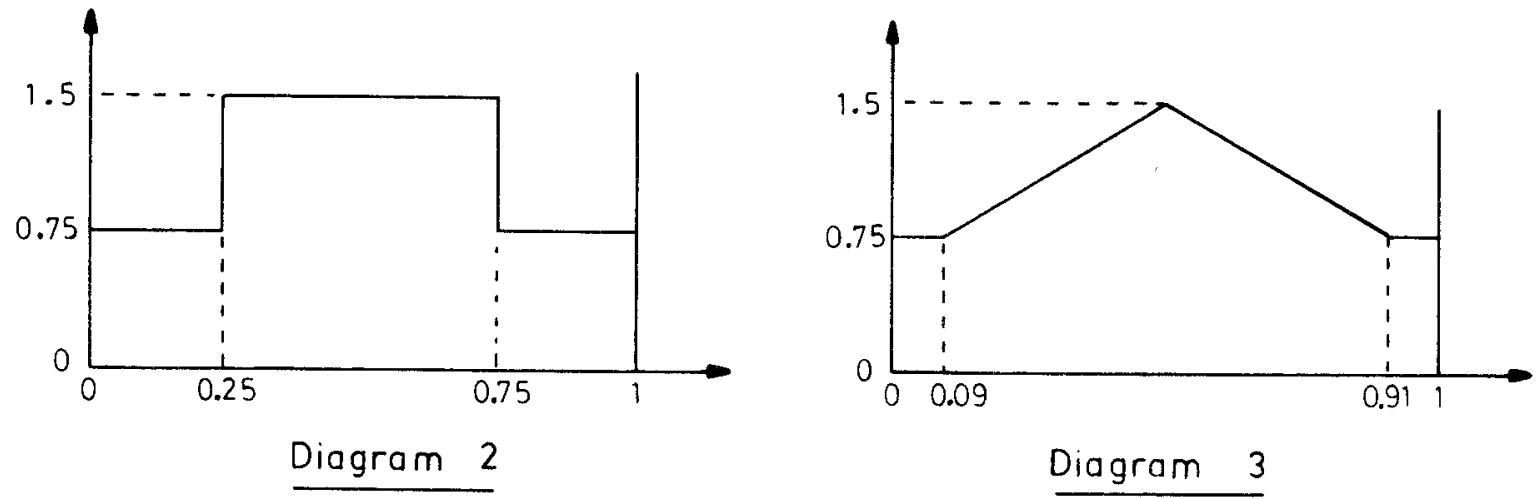


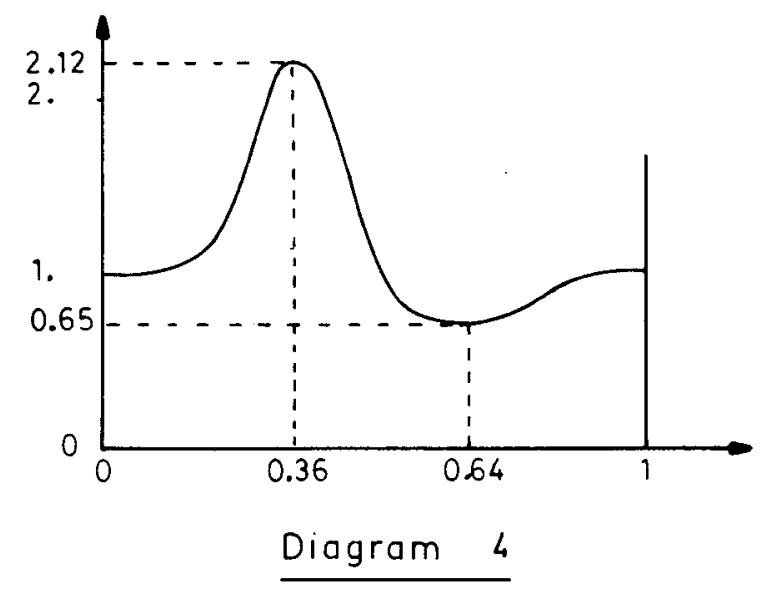

The constants in the definitions of the functions above are chosen such that the eigenvalues of the differential equation are $0, \pm 2 \pi i, \pm 4 \pi i, \ldots$. The function in Diagram 4 is

$$
a(x)=1 /[1-0.33 \sin (2 \pi x)+0.25 \sin (4 \pi x)-0.06 \sin (6 \pi x)] .
$$

The eigenvalues of the matrices are $\pm 2 \pi i$ times the following numbers:

\begin{tabular}{|r|r|r|}
\hline Case 1 & Case 2 & Case 3 \\
\hline 0.0000 & 0.000000 & 0.0000000000 \\
1.0000 & 1.000000 & 1.0000000000 \\
2.0002 & 2.000001 & 2.0000000000 \\
3.0009 & 3.000002 & 3.0000000007 \\
4.0033 & 4.000005 & 4.0000001524 \\
5.0063 & 5.000031 & 5.0000107318 \\
6.0107 & 6.000218 & 6.0003225521 \\
7.0251 & 7.000427 & 7.0046336427 \\
8.0485 & 8.001175 & 8.0338452305 \\
9.0705 & 9.022565 & 9.1417166576 \\
10.5953 & 10.143839 & 10.3759693310 \\
12.7931 & 11.501528 & 11.7242030024 \\
15.2507 & 13.233042 & 13.8615169380 \\
17.8366 & 15.903069 & 19.3309646384 \\
& & \\
\hline
\end{tabular}

According to the estimate in Section 3, it is in all these cases the eigenvalues higher than or equal to $13 /[1 / \mathrm{min} a(x)] \approx 9$ which are expected to be essentially wrong. The smoothness of $a(x)$ influences this number very little, but insstead influences the accuracy of the lower eigenvalues. The figures above may be 
compared to Table 2, where we find the eigenvalues for the classical finite difference approximations in the constant coefficient case. For a fixed mesh with period 27, we can note that the Fourier method with a discontinuous variable coefficient gives an accuracy similar to what the finite difference approximations of orders 6 or 8 give for constant coefficients.

We can also study the influence of aliasing in this way. By aliasing we mean that one high frequency in $\partial u / \partial x$ multiplied with a high frequency in a(x) may get outside the frequency range, and be interpreted by the mesh as a quite different frequency. The Fourier transform is a unitary operator, and thus there corresponds to it a matrix $U$ such that $U^{*} U=I$. The eigenvalues of AS are equal to those of $\left(\mathrm{U}^{*} \mathrm{AU}\right)\left(\mathrm{U}^{*} \mathrm{SU}\right)=\mathrm{A}_{1} \mathrm{~S}_{1}$. Eliminating aliasing corresponds precisely to putting some of the elements of $A_{1}$ to zero. The influence of this is very small. For the three functions above, we get in the aliasing-free case the following eigenvalues:

\begin{tabular}{|r|r|r|}
\hline Case 1 & \multicolumn{1}{|c|}{ Case 2 } & Case 3 \\
\hline 0.0000 & 0.000000 & 0.0000000000 \\
1.0000 & 1.000000 & 1.0000000000 \\
2.0009 & 2.000001 & 2.0000000004 \\
3.0025 & 3.000004 & 3.0000000030 \\
4.0080 & 4.000008 & 4.0000003424 \\
5.0147 & 5.000051 & 5.0000194579 \\
6.0208 & 6.000312 & 6.0005036085 \\
7.0404 & 7.000552 & 7.0063958390 \\
8.0764 & 8.001570 & 8.0420575967 \\
9.1053 & 9.027149 & 9.1629919404 \\
10.7300 & 10.159474 & 10.4118840870 \\
12.9634 & 11.533460 & 11.7626480000 \\
15.4109 & 13.280343 & 13.9989557340 \\
17.9610 & 15.985262 & 19.6778830089 \\
& & \\
\hline
\end{tabular}

If $\mathrm{a}(\mathrm{x})$ somewhere passes zero, we expect to run into trouble with any method, because the analytic solution is then no longer periodic in time. It converges normal1y to a discontinuous function. Neither can we expand the solution in a complete set of eigenfunctions any longer. If $a(x)=\sin x$ we find, instead of bounded eigenfunctions, sets of solutions of the form $e^{-\lambda t}$ (tan $\left.x / 2\right)^{\lambda}$ for any $\lambda$. The matrix AS now gets non-zero real parts in the eigenvalues for all previously considered methods. This problem is however worst for high-order methods. The 
experience is that the Fourier method works well only when $a(x)$ is never close to zero. Otherwise a lower-order method (and a more dense mesh) should be used instead. For eigenvalue evaluations similar to those given above, see Orszag [5].

\subsection{Two-dimensional test example}

In two dimensions we want to approximate

$$
\frac{\partial u}{\partial t}+a(x, y) \frac{\partial u}{\partial x}+b(x, y) \frac{\partial u}{\partial y}=0
$$

with all functions periodic in $\mathrm{x}$ and $\mathrm{y}$. The standard test example for equations of the form (12) is $a(x, y)=-y$ and $b(x, y)=x$ so that the analytic solution is a rotation around origin. It must, however, be pointed out that this example to some extent behaves like a constant coefficient example. The coefficient for $\partial u / \partial x$ does not depend on $x$, and similarly for $y$. Figures 3-8 show test runs for this equation with a cone as initial function. In all these runs, leap-frog with a comparatively small step has been used in the time direction. The size of the time step was the same in all calculations except in the last in Fig. 8, where it had to be divided by 1.5 to maintain stability.

The stability condition for two dimensions is

$\mathrm{S}=\{|\max a(x, y)|+|\max b(x, y)|\} \lambda<a$ constant depending on the method.

$\lambda$ has been chosen such that 400 (or 600) time steps correspond to one revolution for the cone.

\begin{tabular}{|c|l|l|c|c|c|c|c|}
\hline $\begin{array}{c}\text { Figure } \\
\text { number }\end{array}$ & Mesh & $\begin{array}{c}\text { Method } \\
\text { in space }\end{array}$ & $\begin{array}{c}\text { Number } \\
\text { of } \\
\text { revolu- } \\
\text { tions }\end{array}$ & $\begin{array}{c}\text { Number } \\
\text { of } \\
\text { time } \\
\text { steps }\end{array}$ & $\begin{array}{c}\text { Actual } \\
\text { stability } \\
\text { constant } \\
\text { S }\end{array}$ & $\begin{array}{c}\text { Theoretical } \\
\text { stability } \\
\text { limit }\end{array}$ & $\begin{array}{c}\text { Actual } \\
\text { computer } \\
\text { time } \\
(\mathrm{sec})\end{array}$ \\
\hline 3 & $16 \times 16$ & Fourier & 1 & 400 & 0.236 & 0.364 & 25.1 \\
4 & $16 \times 16$ & Fourier & 5 & 2000 & 0.236 & 0.364 & 117.9 \\
5 & $32 \times 32$ & 2nd order & 1 & 400 & 0.487 & 1.000 & 24.0 \\
6 & $64 \times 64$ & 2nd order & 1 & 400 & 0.990 & 1.000 & $(94)$ \\
7 & $32 \times 32$ & 4 th order & 1 & 400 & 0.487 & 0.729 & 38.9 \\
8 & $64 \times 64$ & 4 th order & 1 & 600 & 0.660 & 0.729 & $(232)$ \\
\hline
\end{tabular}

The times for Figs. 7 and 8 are estimated double precision times. To reduce computer time and storage, they were run in single precision (with actual times 68.2 and $176.4 \mathrm{sec}$, respectively). In the diagrams, the paths of the cone were in counter-clockwise direction around the centre of the mesh. For technical details on the test runs (performed on the IBM 370/155 computer at Uppsala University in Sweden) and especially the coding principles for the fast Fourier transform used, the reader is referred to [1]. 
REFERENCES

[1] B. Fornberg, On high order approximations of hyperbolic partial differential equations by a Fourier method", Report No. 39, Apri1 1972, Dept. of Comp. Sci., Uppsala University, Sweden.

[2] B. Fornberg, on the instability of leap-frog and Crank-Nicolson approximations of a non-linear partial differential equation, Math. Comput., 27 (1973), pp. 45-57.

[3] H.-O. Kreiss and J. 01iger, Comparison of accurate methods for the integration of hyperbolic equations, Tellus, 24 (1972), pp. 199-215.

[4] S.A. Orszag, Numerical simulation of incompressible flows within simple boundaries. I. Galerkin (spectra1) representations, Stud. App1. Math. 50 (1971), pp. 293-327.

[5] S.A. Orszag, Comparison of pseudospectral and spectral approximation, Stud. App1. Math. 51 (1972), pp. 253-259.

[6] B. Swartz and B. Wendroff, The relative efficiency of finite difference and finite element methods. I. Hyperbolic problems and splines, Report LA-UR-73-837, 1973, Los Alamos Scientific Laboratory, New Mexico. 


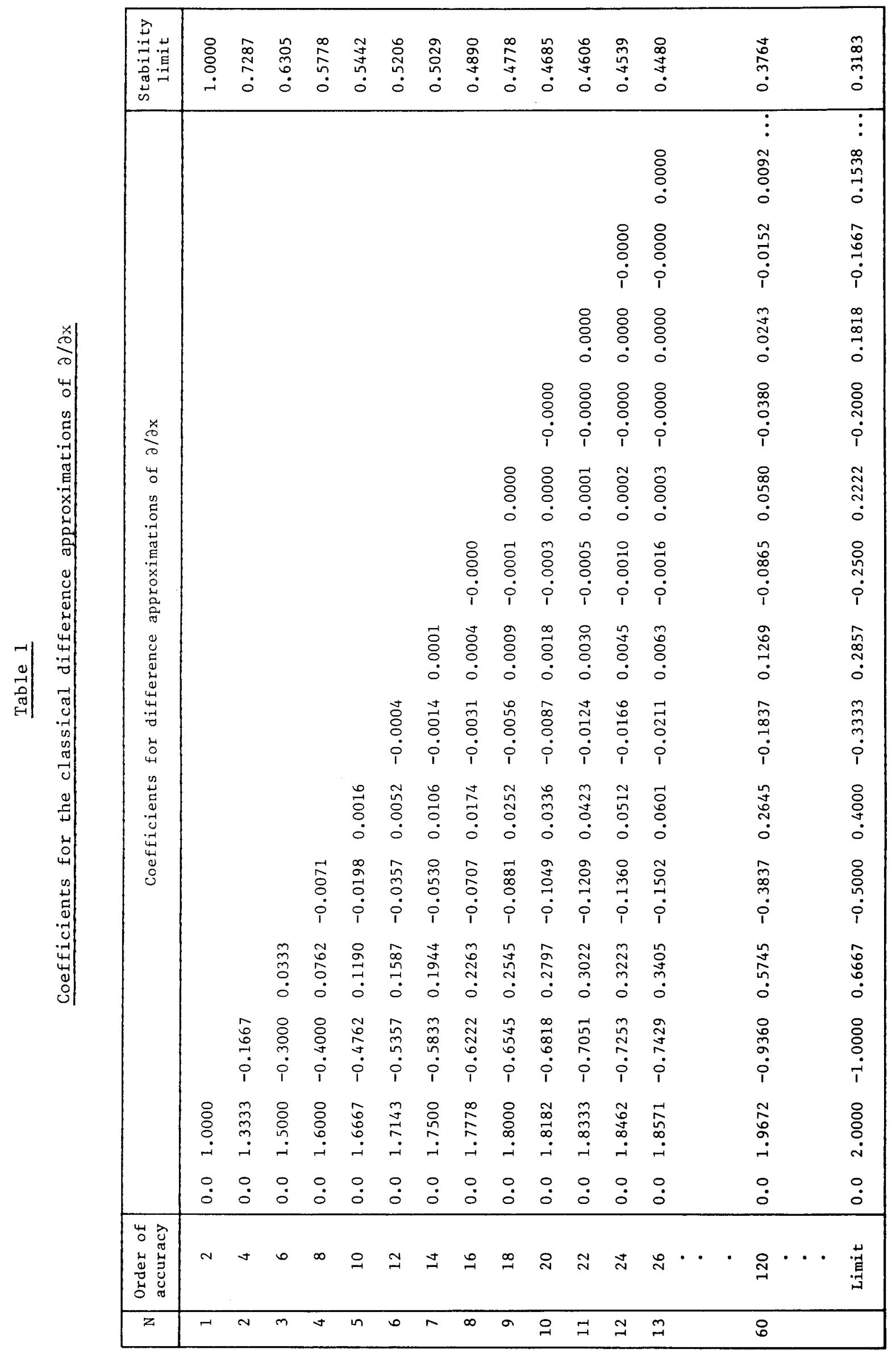




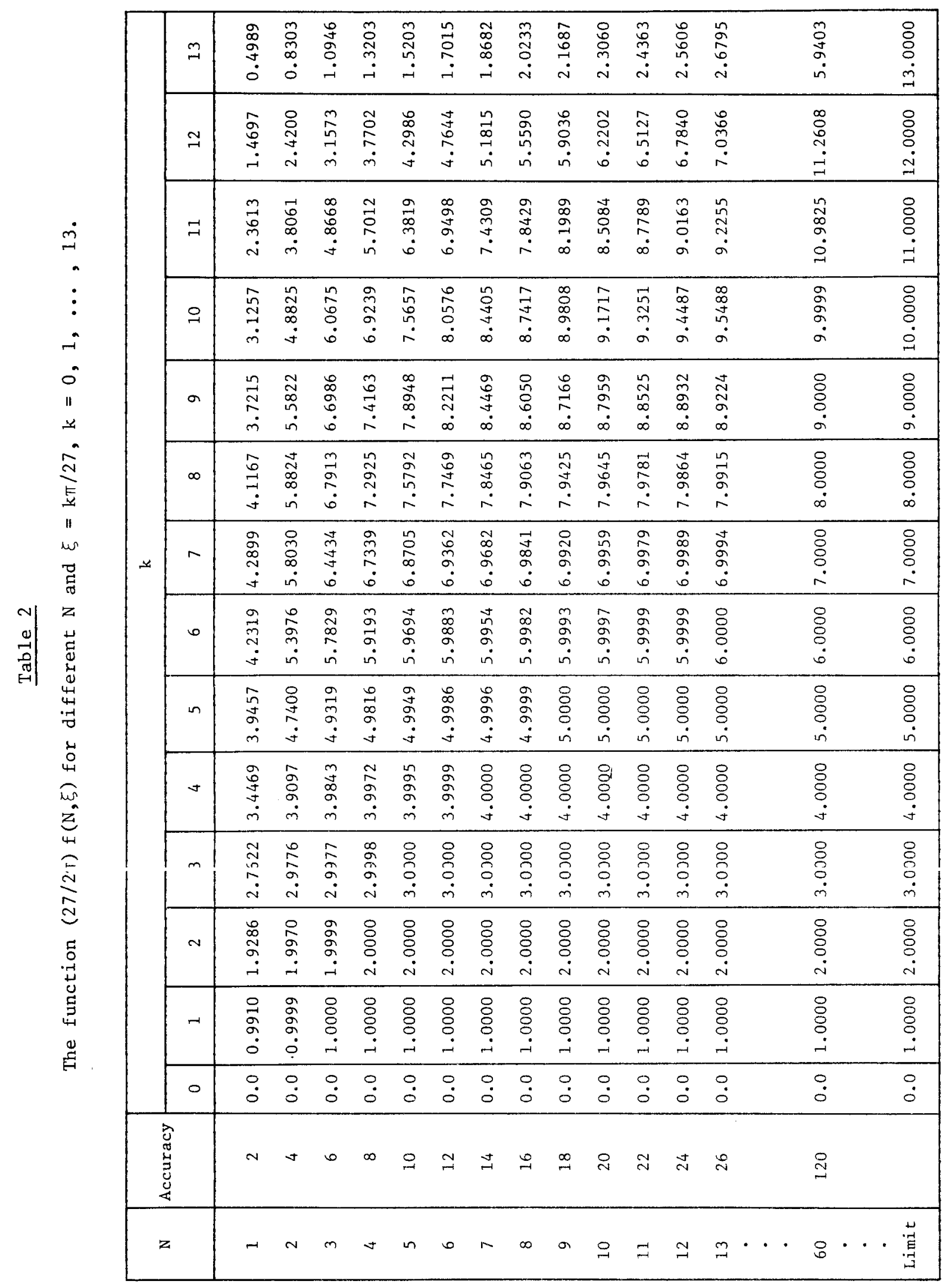




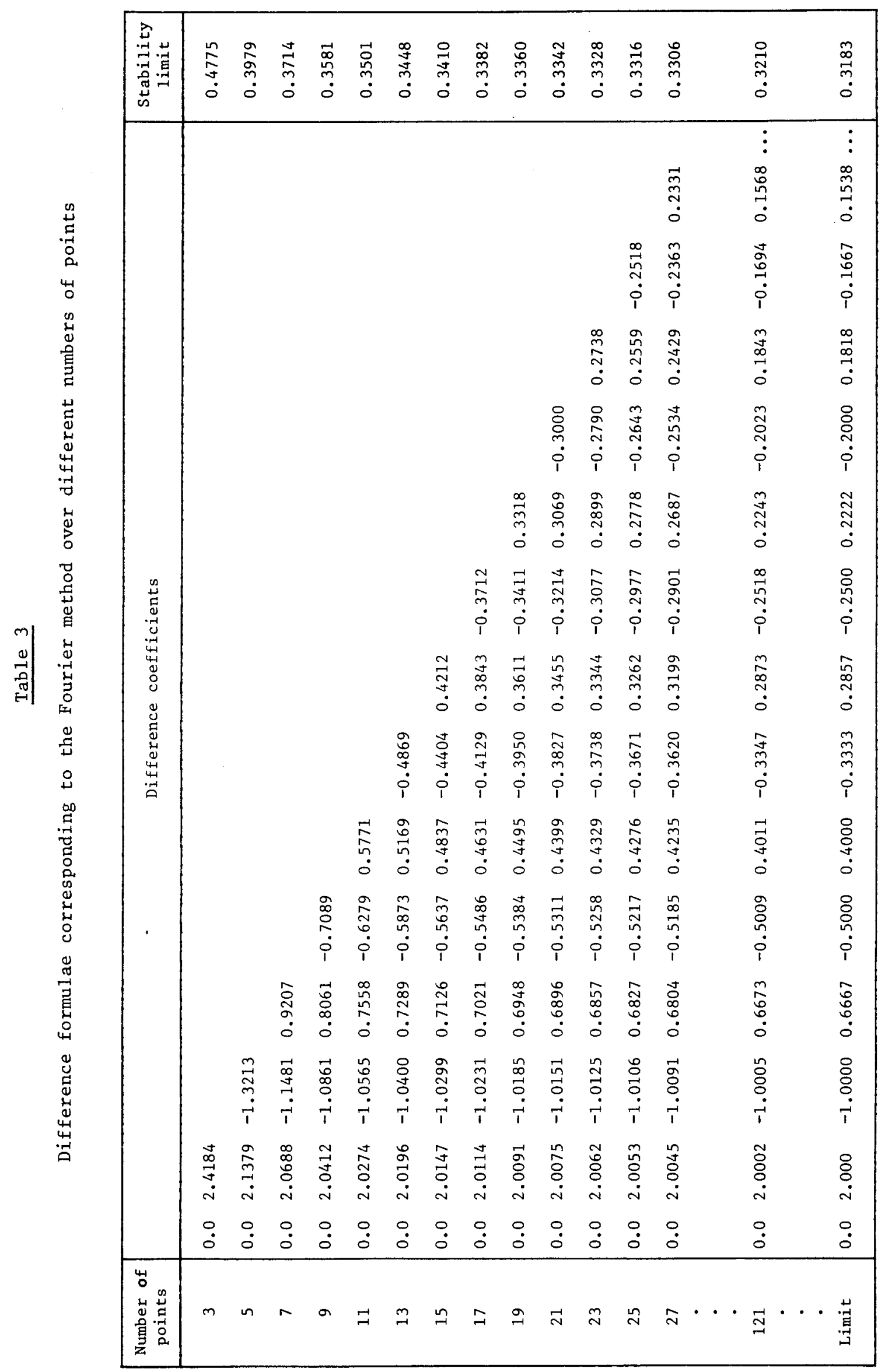




\section{Figure captions}

Fig. 1 : Amplitudes of different frequency components in the 10 th, 30 th and 50 th eigenfunctions of $\partial u / \partial t+a(x) \partial u / \partial x=0$. The function $a(x)$ in diagram 4 is used.

Fig. 2 : Comparison of efficiency of high-order methods.

Fig. 3 : One revolution with the Fourier method. $16 \times 16$ mesh points.

Fig. 4 : Five revolutions with the Fourier method. $16 \times 16$ mesh points.

Fig. 5 : One revolution with the second-order method. $32 \times 32$ mesh points.

Fig. 6 : One revolution with the second-order method. $64 \times 64$ mesh points.

Fig. 7 : One revolution with the fourth-order method. $32 \times 32$ mesh points.

Fig. 8 : One revolution with the fourth-order method. $64 \times 64$ mesh points. 


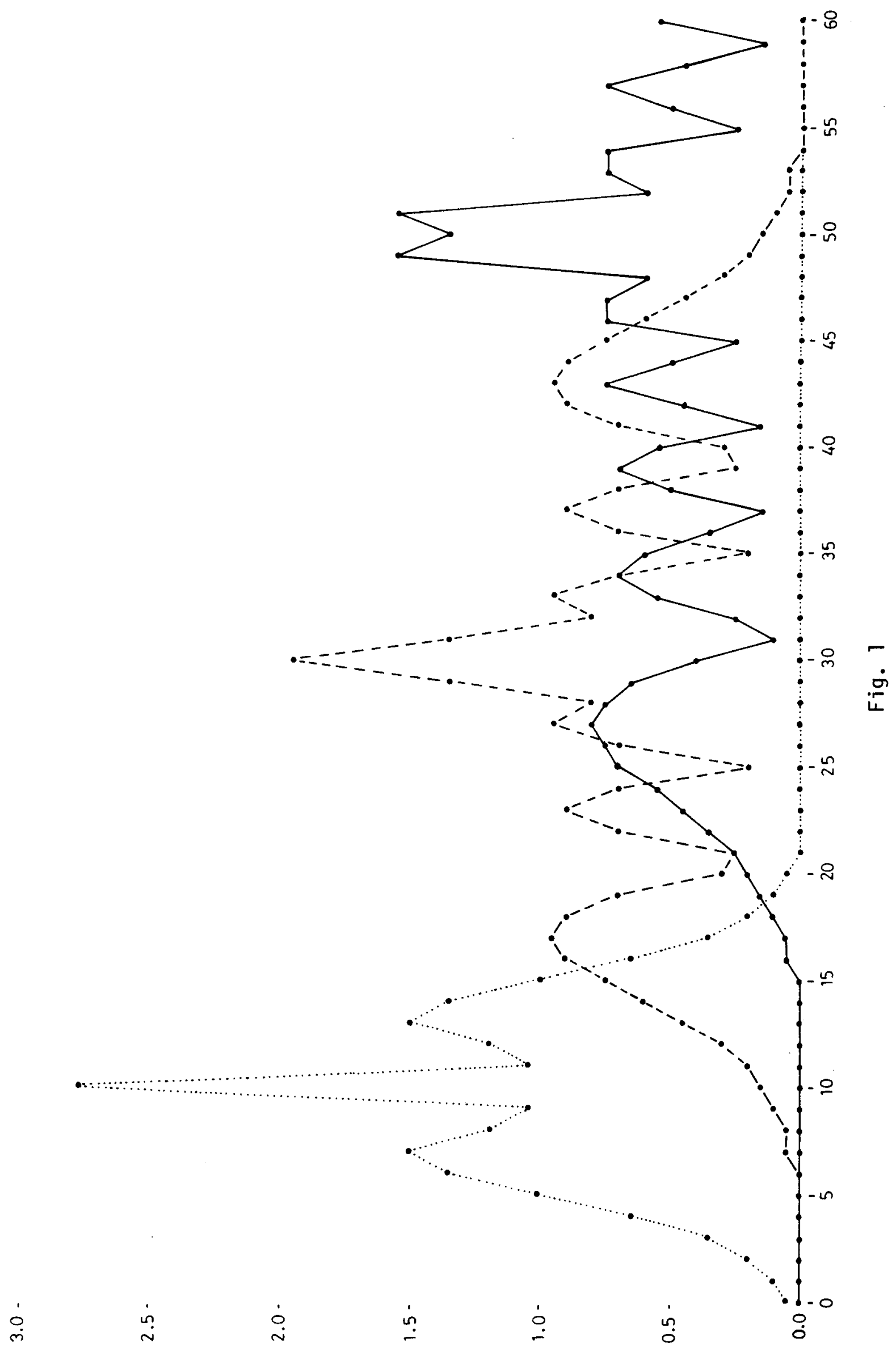




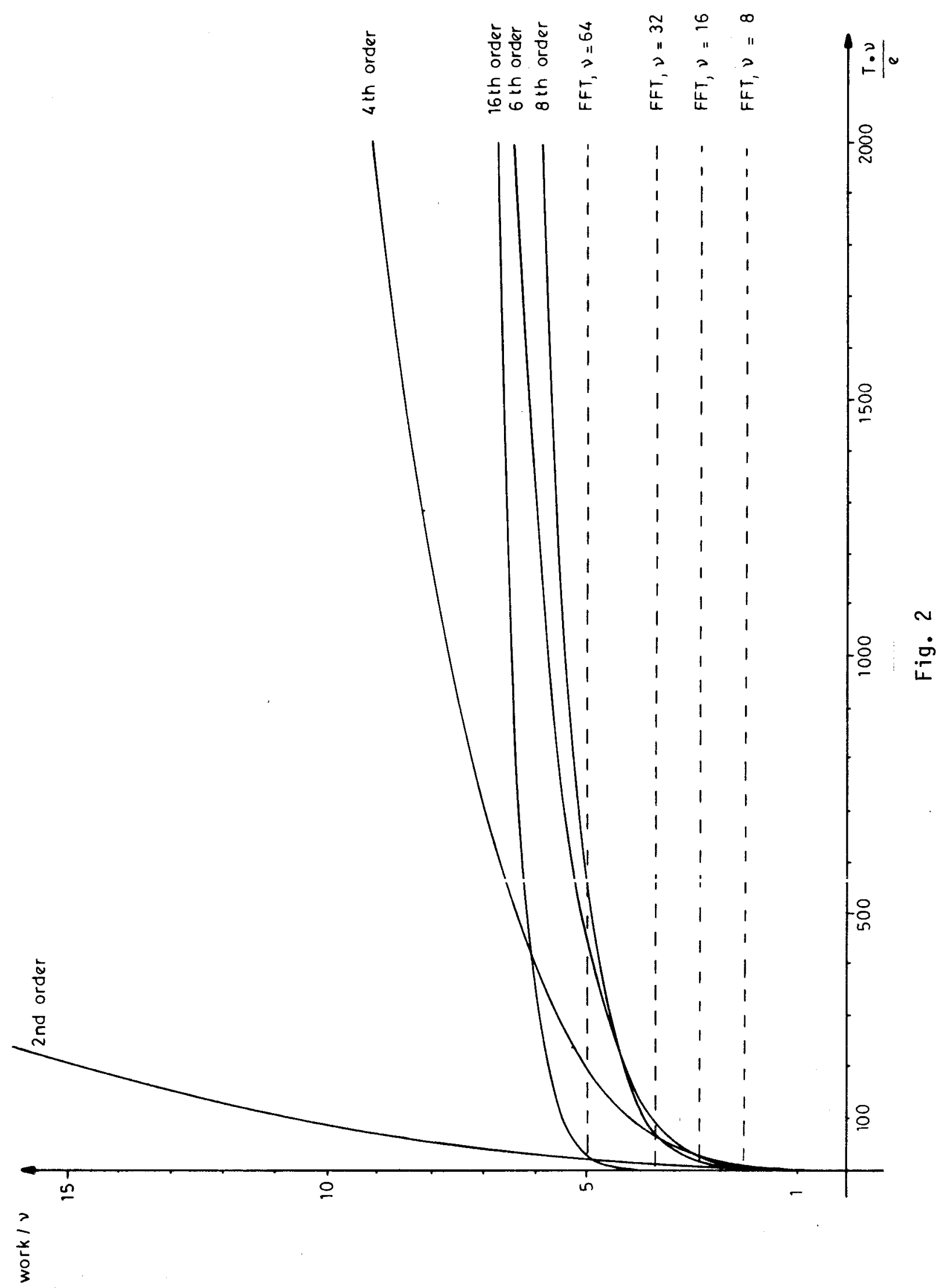




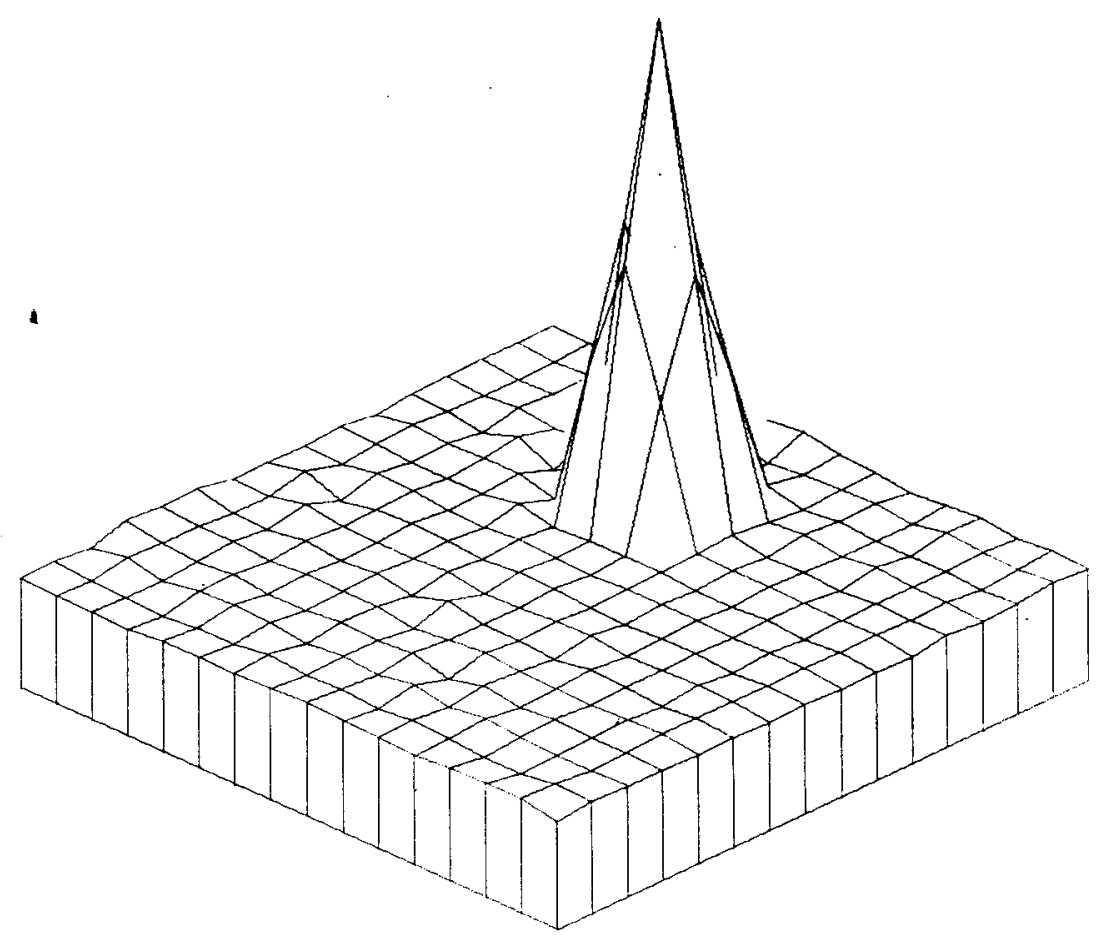

Fig. 3

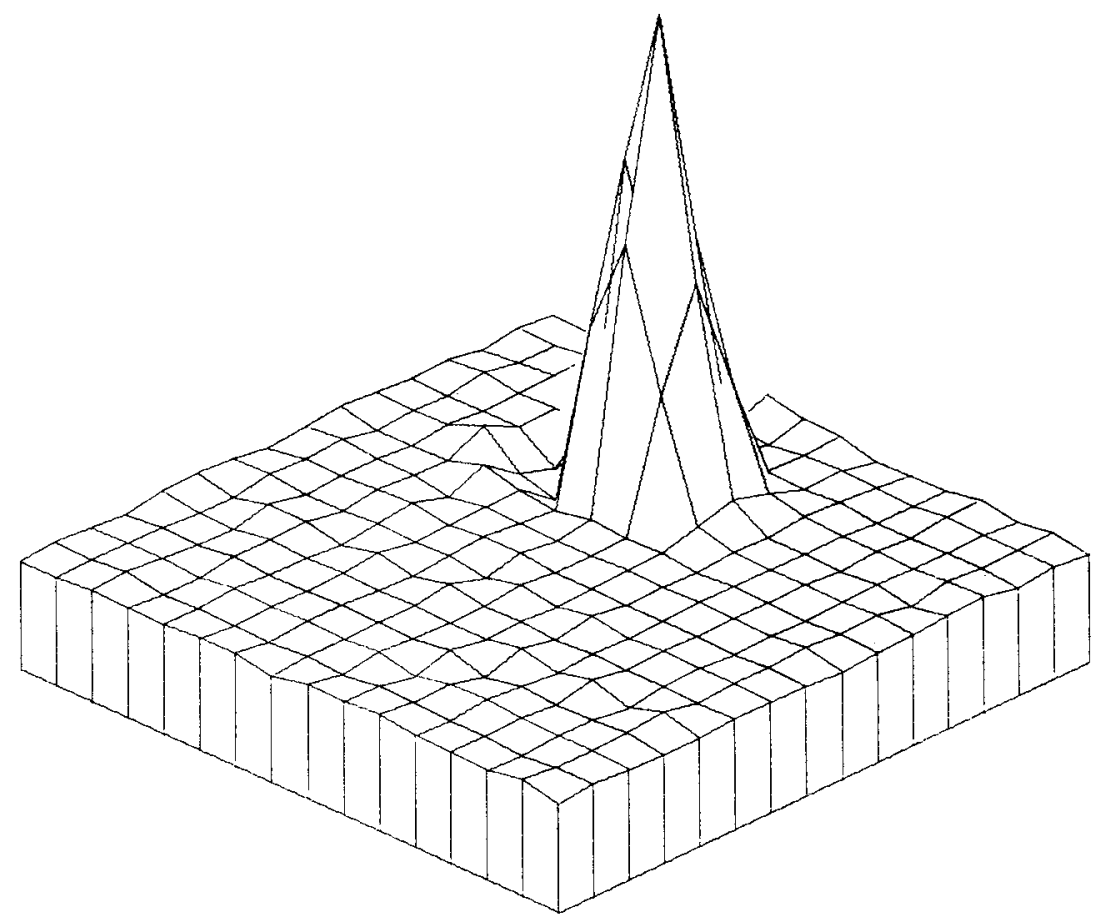

Fig. 4 


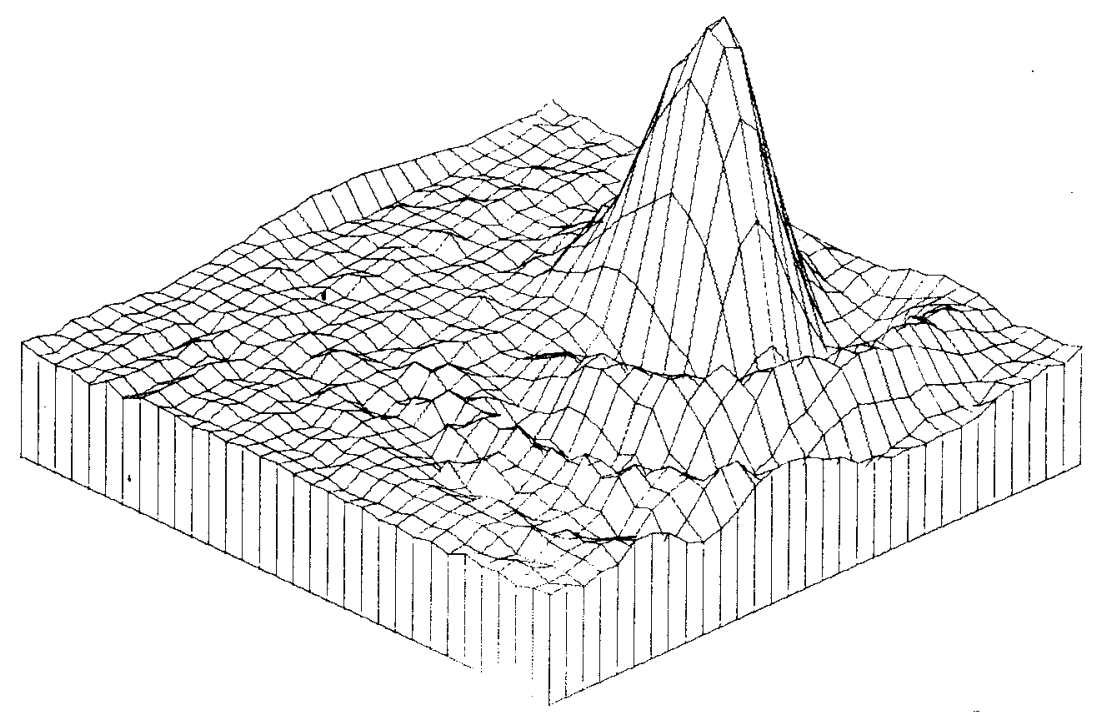

Fig. 5

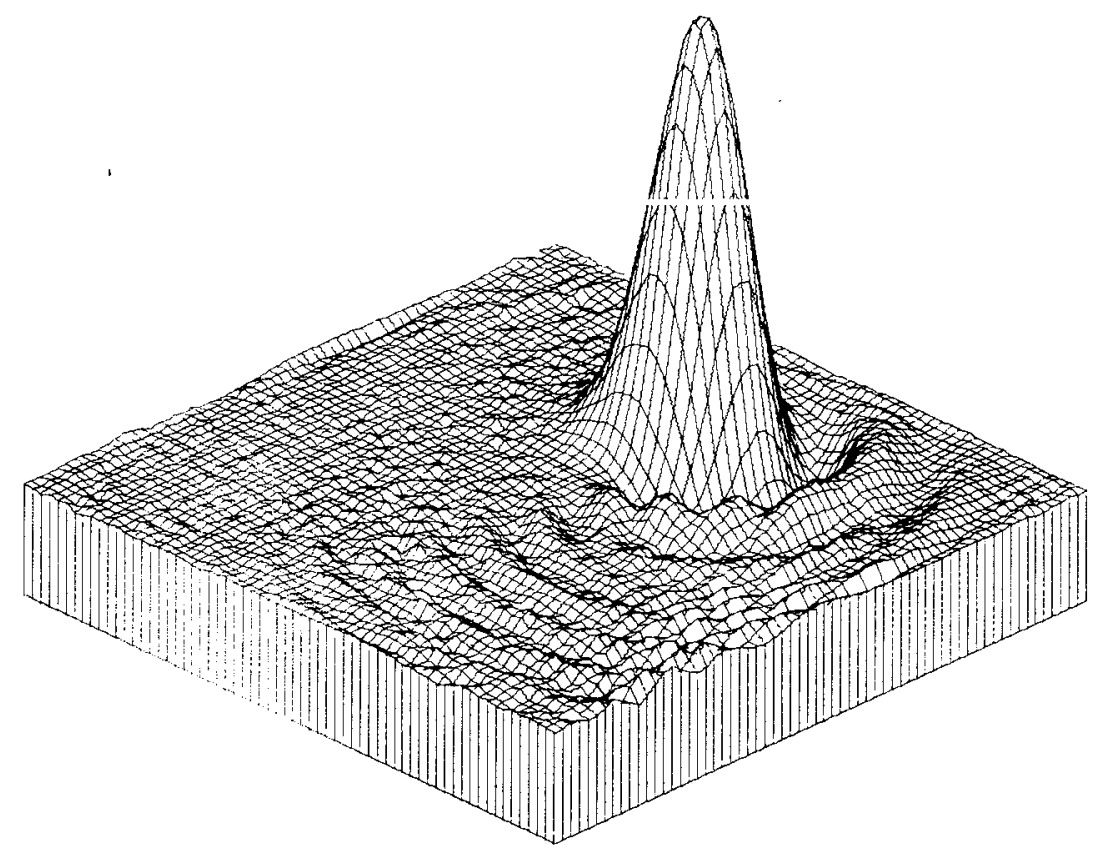

Fig. 6 


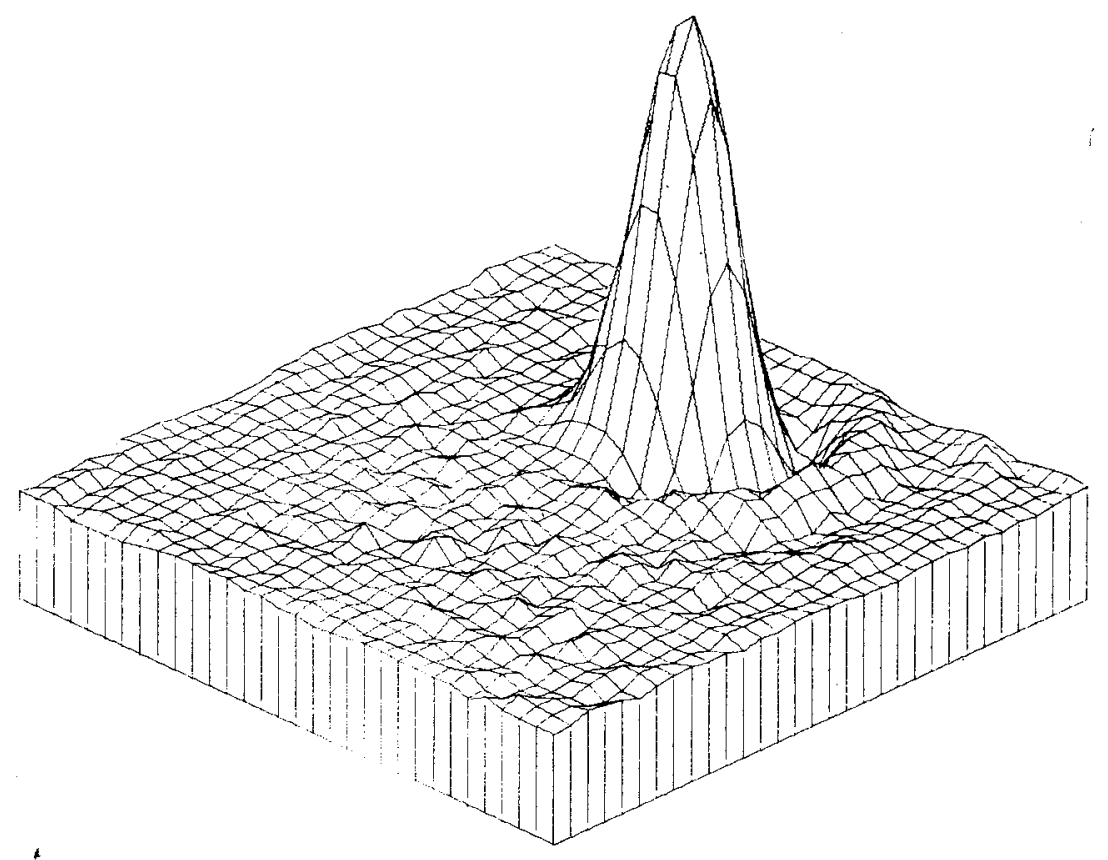

Fig. 7

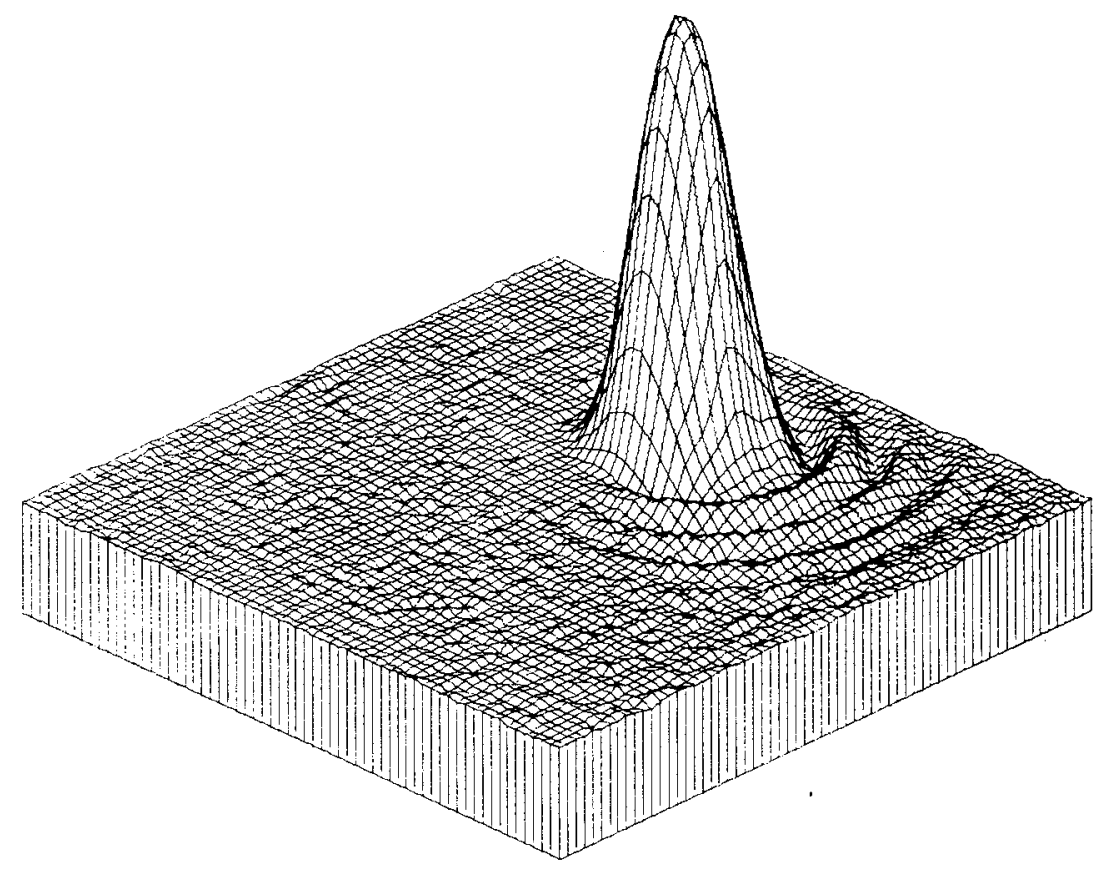

Fig. 8 